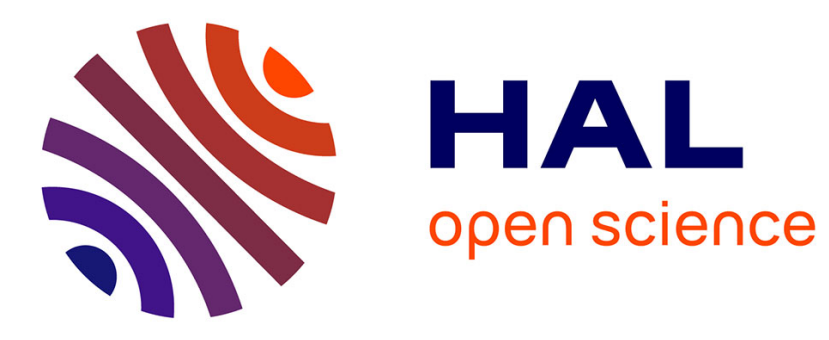

\title{
Partial acute transverse myelitis is a predictor of multiple sclerosis in children
}

\author{
Pierre Meyer, N. Leboucq, N. Molinari, A. Roubertie, M. Carneiro, U. \\ Walther-Louvier, D Cuntz-Shadfar, J Leydet, R Cheminal, G. Cambonie, et \\ al.
}

\section{To cite this version:}

Pierre Meyer, N. Leboucq, N. Molinari, A. Roubertie, M. Carneiro, et al.. Partial acute transverse myelitis is a predictor of multiple sclerosis in children. Multiple Sclerosis Journal, 2014, 20 (11), pp.1485-1493. 10.1177/1352458514526943 . hal-02544667

\section{HAL Id: hal-02544667 \\ https://hal.umontpellier.fr/hal-02544667}

Submitted on 16 Apr 2020

HAL is a multi-disciplinary open access archive for the deposit and dissemination of scientific research documents, whether they are published or not. The documents may come from teaching and research institutions in France or abroad, or from public or private research centers.
L'archive ouverte pluridisciplinaire HAL, est destinée au dépôt et à la diffusion de documents scientifiques de niveau recherche, publiés ou non, émanant des établissements d'enseignement et de recherche français ou étrangers, des laboratoires publics ou privés. 


\title{
Partial acute transverse myelitis is a predictor of multiple sclerosis in children
}

\author{
P Meyer ${ }^{1,2}$, N Leboucq ${ }^{3}, N_{\text {Molinari }}^{4}$, A Roubertie ${ }^{1,5}$, \\ M Carneiro', U Walther-Louvier', D Cuntz-Shadfar ${ }^{1,6}$, \\ J Leydet', R Cheminal', G Cambonie ${ }^{7}$, B Echenne', \\ G Rondouin', K Deiva ${ }^{8,9}$, Y Mikaeloff ${ }^{10,11}$ and F Rivier ${ }^{1,2}$
}

\begin{abstract}
Background: Acute transverse myelitis (ATM) in children is a rare and often severe disease for which there are few known prognostic factors, particularly the subsequent risk of multiple sclerosis (MS) diagnosis.

Objectives: To determine the clinical course and prognostic factors after a first episode of ATM in children.

Methods: Thirty children below 16 years of age diagnosed with a first neurological episode of ATM were included retrospectively. Clinical evaluation, treatment, laboratory, and MRI data were collected.

Results: Median age at onset was II years (range 3-15 years). Follow-up data were available for a median of 4 years (range 0.5-16.7 years). Five patients subsequently had a diagnosis of MS (I7\%), which was associated with acute partial transverse myelitis (odds ratio 5; $95 \%$ confidence interval $2.3-\mathrm{II}$ ), with a $60 \%$ probability of having a relapse at five years $(p<0.0 \mathrm{I})$. The 201 I Verhey criteria correctly identified MS in children with the highest specificity ( $96 \%)$ and sensitivity (80\%).

Conclusion: Acute partial transverse myelitis and brain MRI abnormalities at initial presentation are significantly predictive of a subsequent diagnosis of MS in children with ATM. These findings suggest that closer brain MRI monitoring after acute partial transverse myelitis might make the earlier introduction of disease-modifying therapies possible.
\end{abstract}

\section{Keywords}

Multiple sclerosis, acute transverse myelitis, pediatric, magnetic resonance imaging

\section{Introduction}

Acute transverse myelitis (ATM) is a rare inflammatory process affecting the spinal cord, which manifests as sensory, motor or autonomic dysfunction, with an incidence of 1.71 to 2 per million children. ${ }^{1,2}$ Precise diagnostic criteria for idiopathic and non-idiopathic ATM have been proposed by the Transverse Myelitis Consortium Working Group. ${ }^{3}$

The American Academy of Neurology (AAN) published evidence-based ATM management guidelines in 2011, focusing on two clinical settings: acute complete transverse myelitis (ACTM), defined as acute or subacute inflammation of the spinal cord causing moderate or severe loss of function, and acute partial transverse myelitis (APTM), characterized by incomplete or patchy involvement of at least one spinal segment, with mild to moderate weakness, asymmetric or dissociated sensory symptoms and, occasionally, bladder involvement. ${ }^{4}$ In adult patients, this distinction is considered useful for determining ATM etiology and relapse risk.
'CHRU Montpellier, Neuropédiatrie, France. 2INSERM UI046, Université Montpellier I-2, France. ${ }^{3} \mathrm{CHRU}$ Montpellier, Neuroradiologie, France.

${ }^{4}$ CHRU Montpellier, Service DIM, Université Montpellier I, UMR 729 MISTEA, France.

5INSERM UI05I, Institut de Neurosciences de Montpellier, Université Montpellier I-2, France.

${ }^{6} \mathrm{CHRU}$ Montpellier, Neurophysiologie Clinique, France. ${ }^{7} \mathrm{CHRU}$ Montpellier, Réanimation Pédiatrique, France.

${ }^{8} \mathrm{AP}-\mathrm{HP}, \mathrm{CHU}$ Bicêtre, Neuropédiatrie, Le Kremlin-Bicêtre, France.

${ }^{9}$ National Referral Center for Neuro-Inflammatory Diseases in Children, Le Kremlin-Bicêtre, France.

${ }^{10} \mathrm{AP}-\mathrm{HP}, \mathrm{CHU}$ Bicêtre, Neurosciences, Le Kremlin Bicêtre, France.

'INSERM U669, Université Paris Sud I I, France.

Corresponding author:

Pierre Meyer, Service de Neuropédiatrie, Hôpital Gui de Chauliac, CHRU de Montpellier, 80 avenue Augustin Fliche,

Montpellier Cedex 5, 34295, France.

Email: p-meyer@chu-montpellier.fr 
We hypothesized that this might also be the case in children. We investigated the prognostic factors of childhood ATM, focusing particularly on the risk of multiple sclerosis (MS) diagnosis and disability status at last follow-up.

\section{Method}

\section{Patients}

Inclusion criteria. All children under the age of 16 years evaluated at Montpellier University Hospital (CHRU de Montpellier), France, from January 1994 to December 2009 , with a diagnosis of ATM as a first neurological episode, were included. ATM diagnosis was based on the 2002 criteria. $^{3}$ If spinal magnetic resonance imaging (MRI) showed an appropriately located high signal-intensity lesion on T2-weighted sequences but no clear cut enhancement of the abnormality following gadolinium administration and no CSF abnormality, patients were considered to have 'possible ATM' and were included. ${ }^{3}$

Exclusion criteria. Patients with a previous neurological history, systemic or metabolic disease, history of previous spinal radiation, extra-axial spine compression or thrombosis of the anterior spinal artery on MRI were excluded.

\section{Standard protocol approvals, registrations, and patient consent}

The parents of the patients gave written informed consent to care. The procedures described herein are all part of routine care on our hospital. The study has been submitted to the ethics committee of Montpellier University Hospital: this observational and retrospective study did not require approval of the institutional review board and no informed written consent was required, in line with French law.

\section{Data collection}

All the information collected was coded to protect confidentiality.

Clinical data. Retrospective clinical data during acute phase were collected from our hospital database in a standardized manner: demographic, personal, and familial history of MS and other autoimmune disease, factors preceding ATM, such as vaccination, trauma, or infection. The acute illness description included first symptoms, time to diagnosis and maximal clinical expression, and plateau duration. Patient management, particularly the need of bladder catheterization, and the type of treatment were also recorded.

Walking ability during the acute phase was assessed with a modified version of the Hughes Functional Disability Scale (HFDS) previously used in Guillain-Barré syndrome, ${ }^{5}$ and in the largest published pediatric ATM series: ${ }^{6}$
$0=$ normal, $1=$ minor symptoms, fully capable of manual work, 2 = able to walk more than 30 feet without assistance, $3=$ able to walk more than 30 feet with assistance, $4=$ bedbound/wheelchair-bound, $5=$ requiring assisted ventilation, $6=$ dead.

Laboratory and MRI data. We collected the results of cerebrospinal fluid (CSF) tests, including white blood cell (WBC) count, biochemistry findings, and oligoclonal band detection. Serum samples were tested for 19 infectious pathogens, with convalescent titers for the same pathogens determined within six weeks of the acute phase, when possible. An infectious disease was defined by two recorded oral temperatures above $38.5^{\circ} \mathrm{C}\left(101.3^{\circ} \mathrm{F}\right)$, two recorded serum WBC elevations above 11,000 cells $/ \mathrm{mm} 3$, or positive PCR or IgM converting to IgG against a specific pathogen.

Magnetic resonance images were acquired on $1.5 \mathrm{~T}$ scanners. MRI data included scans of the spine and brain at the same time during the acute phase and follow-up: localization, extent and type of spine lesions, gadolinium enhancement on T1-weighted images (T1W). Patients with lesions at least three segments long were classified as having 'longitudinally extending transverse myelitis' (LETM). ${ }^{7}$ MRI brain images were examined for the presence of T2-weighted (T2W) lesions (supratentorial, infratentorial, basal ganglia/thalamus) and were classified according to: (1) the 2010 McDonald dissemination in space and time criteria; ${ }^{8}(2)$ the 2011 Verhey criteria; ${ }^{9}$ (3) the KIDMUS criteria; ${ }^{10}(4)$ the CHAMPS criteria. ${ }^{11}$ Criteria definitions are shown in Table 1.

All baseline images were reviewed retrospectively, with a standardized MRI record form, by an experienced neuroradiologist (NL) and neuropediatrician (FR) blinded to clinical symptoms, disease progression, and initial MRI analysis, to obtain a consensus. Visual evoked potentials (VEPs) were also considered, when performed.

Outcome data. Outcome data were collected from our hospital database for patients having ongoing care provided by our department, or from physicians caring for other children in the community. Outcomes included subsequent diagnosis of MS and Kurtzke Expanded Disability Status Scale (EDSS) score at last follow-up, focusing on scores $\geq 4$, defined as limited walking ability but able to walk for more than $500 \mathrm{~m}$ without assistance or resting. ${ }^{12}$

\section{Definitions and classification}

Patients with incomplete or patchy involvement of at least one spinal segment, with mild to moderate weakness, asymmetric or dissociated sensory symptoms and, occasionally, bladder involvement were defined as having APTM. ${ }^{4}$ Patients with symmetric, moderate or severe loss of function due to a spinal cord lesion were considered to have ACTM. 


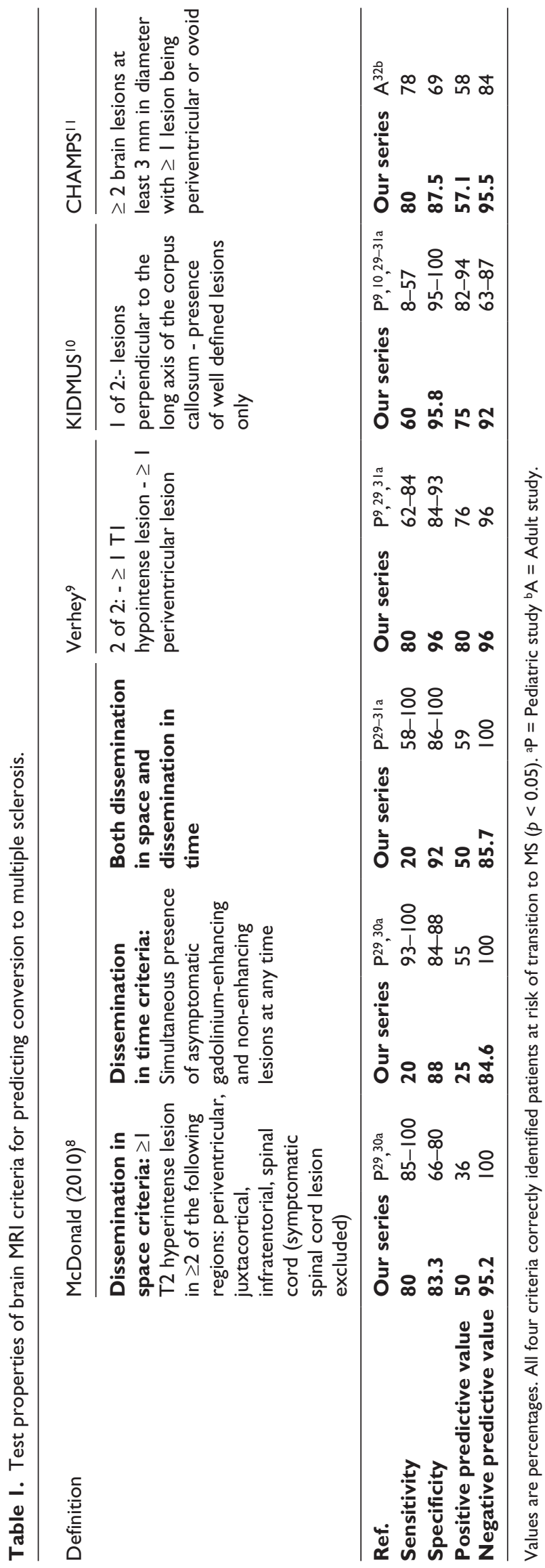

Diagnosis at presentation and most recent assessment was classified as: isolated transverse myelitis, i.e. clinicoradiological TM with no clinical brain disease and normal brain MRI; clinically isolated syndrome (CIS), i.e. clinically isolated ATM with abnormal brain MRI but no encephalopathy; polyfocal CIS, i.e. multifocal neurological deficit including ATM, without encephalopathy; acute disseminated encephalomyelitis (ADEM), i.e. clinical ATM with encephalopathy; ${ }^{13}$ or MS fulfilling the 2010 McDonald criteria. $^{8}$

\section{Statistical analysis}

Descriptive data were compared in $\chi^{2}$ tests or Fisher's exact tests for proportions, and t-tests or Wilcoxon tests for continuous measures. Kaplan-Meier curves were plotted and the significance of differences was assessed in log-rank tests. Statistical significance was reached with a $p$ value of less than 0.05 . We were unable to conduct multivariate analysis due to the small size of our population.

\section{Results}

\section{General overview}

In total, 30 patients with acute transverse myelitis before the age of 16 years were included in the study, with a mean follow-up of $5.1 \pm 4.4$ years (median: 4 years, range: $0.5-$ 16.7 years). Median age at onset was 11 years (range 3-15 years), but was lower in cases of ATM following infection (7.7 vs 12.7 years, $p<0.01$ ). The female:male ratio was $1.13: 1$ and $47 \%$ of cases (14/30) occurred during the winter. The main clinical, CSF, and MRI characteristics are shown in Table 2.

At first presentation, 53\% (16/30) had isolated ATM, $17 \%(5 / 30)$ had monofocal CIS, 17\% (5/30) had ADEM, $13 \%(4 / 30)$ had polyfocal CIS, and none had MS according to the $2010 \mathrm{McDonald}$ criteria. At most recent assessment, five of the 30 patients were diagnosed with MS fulfilling the 2010 McDonald criteria (see Figure 1).

\section{Descriptive data (Table 2)}

Clinical characteristics. In $60 \%$ (18/30) of cases, neurologic manifestations developed after an infection occurring a median of 6 days (range 2-28 days) before the onset of neurologic symptoms: isolated fever (7/18), otolaryngologic infection (6/18), gastroenteritis (2/18), influenza-like illness (2/18), pneumonia (1/18), or measles (1/18). No patient had been immunized or had an allergy shot within 30 days of the first symptoms. All patients were investigated for infectious agents in blood and CSF at ATM onset, and 67\% (20/30) underwent serological testing after three to six weeks. Minor trauma (fall or twist) up to one day before ATM onset was described in $17 \%$ of cases (5/30). Clinical findings (no clear arterial distribution clinical 
Table 2. Main clinical, CSF, and MRI characteristics of the patients during the acute phase.

\begin{tabular}{|c|c|c|c|c|c|c|}
\hline \multirow[t]{3}{*}{ Clinical data } & \multirow{3}{*}{$\begin{array}{l}\text { Total } \\
n=30\end{array}$} & \multicolumn{5}{|c|}{ Diagnosis at the end of follow-up } \\
\hline & & \multirow{2}{*}{$\begin{array}{l}\text { Isolated ATM } \\
n=14\end{array}$} & \multirow{2}{*}{$\frac{\text { CIS }}{n=5}$} & \multirow{2}{*}{$\frac{M S}{n=5}$} & \multirow{2}{*}{$\begin{array}{l}\text { ADEM } \\
n=4\end{array}$} & \multirow{2}{*}{$\frac{\text { Other* }}{n=1}$} \\
\hline & & & & & & \\
\hline Median age at onset [y] (range) & II (3-15) & $12(3-15)$ & $5(3-14)$ & $13(6-15)$ & $10.5(6-11)$ & 4 \\
\hline Preceding infection (\%) & 60 & 53 & 100 & 20 & 75 & 100 \\
\hline $\begin{array}{l}\text { Median duration to maximal } \\
\text { functional symptoms [d] } \\
\text { (range) }\end{array}$ & $5(1-17)$ & $3(1-12)$ & $6(3-7)$ & $5(4-5)$ & $6(2-12)$ & 17 \\
\hline Pain (\%) & 67 & 71 & 100 & 40 & 50 & 100 \\
\hline Fever $(\%)$ & 40 & 40 & 60 & 20 & 25 & 100 \\
\hline Complete paraplegia (\%) & 46 & 53 & 40 & 20 & 75 & 100 \\
\hline Pyramidal signs (\%) & 50 & 47 & 40 & 75 & 50 & 0 \\
\hline Sensory loss (\%) & 60 & 79 & 40 & 60 & 25 & 100 \\
\hline Sphincter deficit (\%) & 77 & 47 & 20 & 20 & 25 & 0 \\
\hline Bladder catheterization (\%) & 47 & 71 & 40 & 20 & 25 & 0 \\
\hline APTM (\%) & 33 & 21 & 40 & $100 \ddagger$ & 0 & 0 \\
\hline Median HFDS score (range) & $3.5(I-4)$ & $4(I-4)$ & $3(2-4)$ & $3(2-4)$ & $4(3-4)$ & 4 \\
\hline $\begin{array}{l}\text { Median CSF WBC count } \\
{\left[\text { cells } / \mathrm{mm}^{3}\right] \text { (range) }}\end{array}$ & $15.5(0-700)$ & $10(0-700)$ & $30(2-76)$ & II (2-64) & $200(30-530)$ & 20 \\
\hline $\begin{array}{l}\text { Median CSF protein } \\
\text { concentration }[\mathrm{g} / \mathrm{l}]\end{array}$ & $0.45(0.15-1.79)$ & $0.39(0.21-0.89)$ & $0.38(0.15-0.79)$ & $0.44(0.2 \mathrm{I}-0.67)$ & $0.69(0.42-1.79)$ & 0.58 \\
\hline $\begin{array}{l}\text { Median lesion length on spinal } \\
\text { MRI [segment] (range) }\end{array}$ & $5(0-15)$ & $3(0-15)$ & $10(4-16)$ & $4(0-9)$ & $6(0-7)$ & 20 \\
\hline Abnormal brain MRI (\%) & 45 & 0 & 100 & 80 & 100 & 0 \\
\hline
\end{tabular}

*Final diagnosis of connective tissue disorder; $¥$ Significantly higher in MS than in the non-MS population; đSignificantly lower in MS than in the non-MS population. ATM = acute transverse myelitis; APTM = acute partial transverse myelitis; CIS = clinically isolated syndrome; $M$ SS = multiple sclerosis; $\mathrm{ADEM}=$ acute disseminated encephalomyelitis; $\mathrm{CSF}=$ cerebrospinal fluid; WBC = white blood cell; HFDS = Hughes Functional Disease Scale.

deficit consistent with thrombosis of the anterior spinal artery, time to reach maximal severity $>4$ hours from onset) and spinal MRI findings in these five patients were not consistent with infarction.

The main clinical characteristics are shown in Table 2. Acute partial transverse myelitis was present in $33 \%$ of the patients (10/30). By definition, the five patients initially diagnosed with ADEM had encephalopathy during the acute phase. ${ }^{13}$

CSF and MRI characteristics. Cerebrospinal fluid (CSF) was obtained by lumbar puncture, 1 to 13 days after symptom onset (median 2.5 days). Abnormalities were found in 17 patients (57\%, see Table 2$)$. CSF oligoclonal bands were detected in 4 of 16 patients tested ( 3 MS and 1 postinfectious polyfocal CIS) and were not significantly associated with MS.

All patients underwent a first cerebral and spinal MRI between 1 and 11 days after symptom onset (median: 4 days) and images were available for 29 patients. Spinal MRI was abnormal in 93\% of cases (27/29): T2-weighted (T2W) signal abnormalities were identified at cervical level in $55 \%$ patients (16/29), thoracic level in 72\% (21/29) and lumbosacral level in 24\% (7/29). Multifocal lesions were detected in $26 \%$ of patients $(8 / 29)$ and hypointense lesions on T1-weighted (T1W) sequences were found in two patients. LETM was found in 20 of 29 patients (69\%). Total median segment length was 5 segments (range 0-15 segments). Lesion count did not differ significantly between patients with and without MS $(p=0.283)$. Gadolinium infusion demonstrated lesion enhancement on T1W images in $31 \%(9 / 29)$ of cases. Both patients who had a first normal spinal MRI showed lesions on T2W sequences without gadolinium enhancement on a second scan performed 5 and 7 days later.

Cerebral MRI evidence of white matter lesions was obtained in $45 \%(13 / 29)$ of cases: the hypersignal areas on T2W images were supratentorial in $38 \%(11 / 29)$ of cases, infratentorial in $31 \%(9 / 29)$, and in the basal ganglia/thalamus in $28 \%(8 / 29)$ of cases. One patient (final diagnosis: MS) had a T2W hypersignal on optic nerves. The test properties of the 2010 McDonald, Verhey, KIDMUS, and CHAMPS criteria are shown in Table 1.

Visual evoked potentials (VEP) and immunochemistry. VEP was carried out during the acute phase in $60 \%$ of patients $(18 / 30)$ and optic neuritis was found in $13 \%$ of cases $(4 / 30)$. Serum anti-aquaporin $4 \mathrm{IgG}$ was absent in all four children 


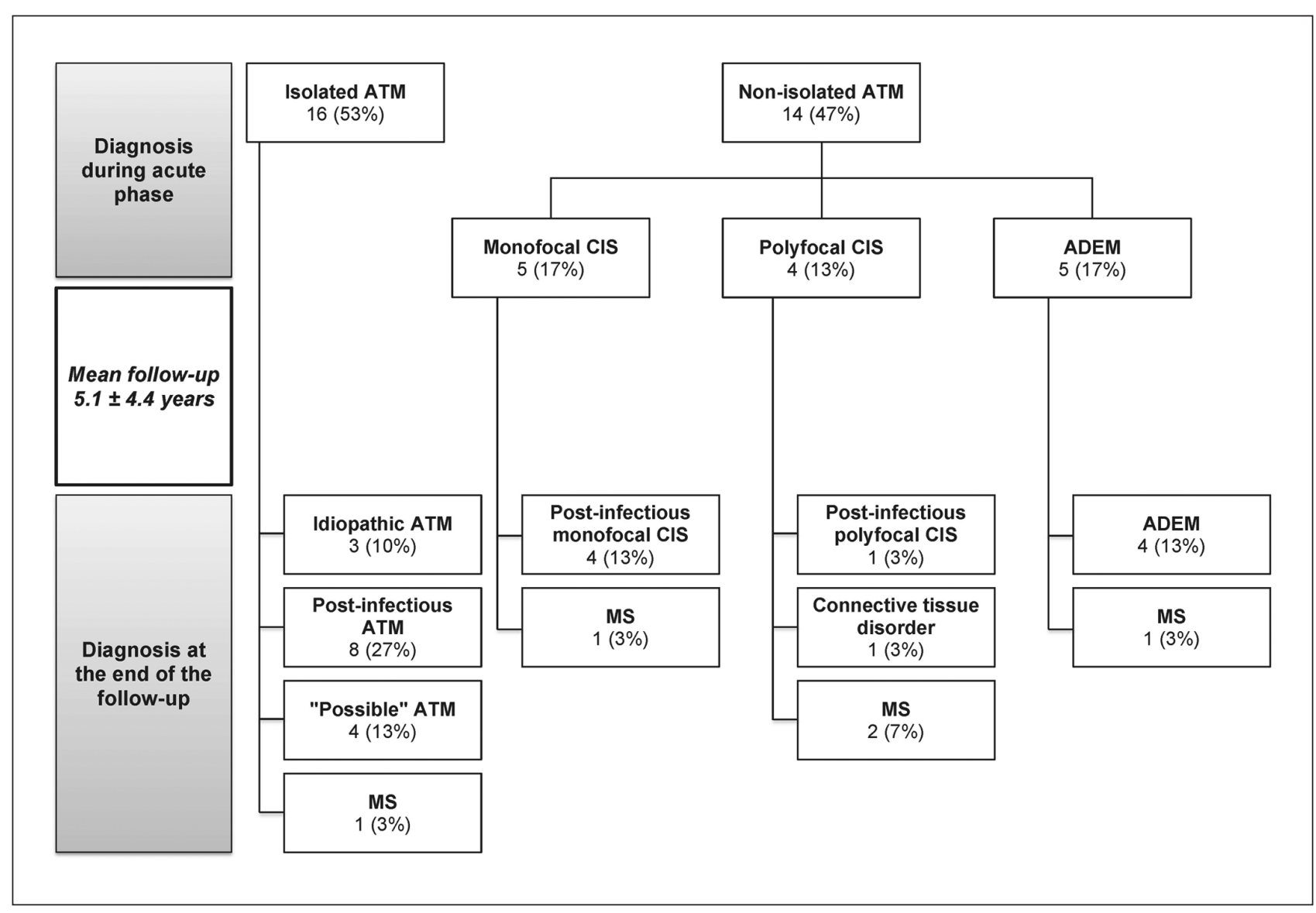

Figure I. Patient diagnosis flow chart. ATM = acute transverse myelitis; 'possible' ATM = clinical ATM with T2 hyperintensity on spinal MRI without gadolinium enhancement, and normal CSF; ${ }^{3} \mathrm{CIS}=$ clinically isolated syndrome; monofocal CIS = ATM with abnormal brain MRI, without encephalopathy; polyfocal CIS = multifocal neurological deficit including ATM, without encephalopathy; $\mathrm{ADEM}=$ acute demyelinating encephalomyelitis; $\mathrm{MS}$ = multiple sclerosis.

tested: only 1 of 4 had optic neuritis, with LETM and a subsequent diagnosis of MS. None of the patients presented serologic evidence of connective tissue disease at presentation but, during follow-up, one patient displayed antinuclear antibodies, lupus anticoagulant and ANCA, suggestive of this condition.

Type of treatment. All patients were prescribed treatment at ATM presentation: $87 \%$ (26/30) received high-dose IV methylprednisolone, 50\% (15/30) received IV immunoglobulin, and both were prescribed in $37 \%$ of cases $(11 / 30)$. Mean interval between treatment beginning and MRI was of 0.5 days (median: 0 days, range: 4 days before MRI to 5 days after first MRI). We found no correlation between type of treatment and outcome.

\section{Prognosis factors}

Follow-up duration was $5.1 \pm 4.4$ years (range: $0.5-16.7$ ) and exceeded one year in more than $96 \%$ of cases $(29 / 30)$.
Most (80\%) patients (24/30) were able to walk independently within one month of onset. The other six patients who were unable to walk independently within one month of onset had a median HFDS score of 4 during acute phase (range: $3-4$ ), a median plateau duration phase of 10 days (range: 5-20 days), and a median lesion length of 6 segments on spinal MRI (range: 2-15 segments). Outcome was not related to seasonality, sex, age at onset, time to maximal symptom expression, fever, pain, pyramidal signs at the time of presentation, CSF findings or spinal MRI characteristics during acute phase.

Diagnosis of MS. Acute partial transverse myelitis at initial presentation was a significant prognostic factor for subsequent MS diagnosis (odds ratio [OR] 5, 95\% confidence interval $[\mathrm{CI}] 2.3-1, p<0.01)$ with a $40 \%$ probability of being relapse-free at five years ( $p<0.01$, see Figure 2). Higher acute-phase HDFS score (3 or 4 ) was associated with significant protection against diagnosis of MS (OR $0.048,95 \%$ CI $0.01-0.54, p<0.05$ ). 


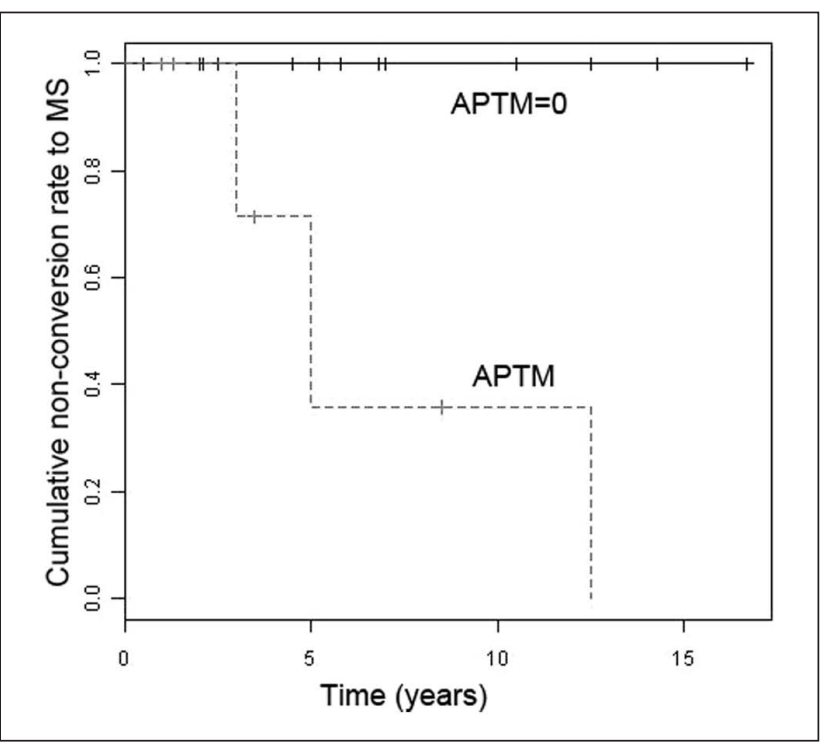

Figure 2. Acute partial transverse myelitis and conversion to multiple sclerosis. Survival over time to conversion to MS, according to the presence of APTM during the acute phase ( $p<$ 0.01 , in a log-rank test). All 5 MS patients had APTM, whereas only $5 / 25$ of non-MS patients had APTM $(P<0.01)$. APTM $=$ acute partial transverse myelitis; $M S=$ multiple sclerosis.

The 2010 McDonald dissemination in space, Verhey, KIDMUS, and CHAMPS criteria correctly identified patients at risk of MS diagnosis $(p<0.05$; see Table 1).

Disability scale score at last follow-up assessment (EDSS). EDSS score at last follow-up was significantly higher in patients taking more than one month to walk independently (mean of 4.3 vs. $0.9, p<0.01$ ) and in cases of complete paraplegia during acute phase (mean 2.3 vs. $0.8, p<0.05$ ). The need for urinary catheterization during acute phase was significantly associated with a final EDSS score $\geq 4$ (OR 2.5, 95\% CI $1.5-4, p<0.05)$. Patients with idiopathic ATM were more likely to have an EDSS score $\geq 4$ at one year (OR 24, 95\% CI 1.5-395, $p<0.05$ ), but this difference was not significant at the end of follow-up.

\section{Discussion}

This retrospective series of children below the age of 16 years with single episodes of ATM at a single academic center included 30 patients over a 15 -year period. Previous series specifically studying pediatric ATM concerned eight to 47 patients included over four to 25 years. Apart from one, ${ }^{2}$ all were monocentric and retrospective, ${ }^{6,14-18}$ and not all used the 2002 diagnostic criteria. ${ }^{6}, 14$ Other larger studies of pediatric cases of acute CNS inflammatory demyelination did not include details of clinical symptoms at presentation. ${ }^{1,10,19}$ It is difficult to compare our results with those of previously published series, as the 2002 criteria (including markers of spinal inflammation) were not used in five of these studies. The clinical presentation and course of ATM in our patients was similar to those in previous pediatric series, in terms of age at presentation, initial course and preceding infection (see Table 3). Surprisingly, none of our patients was under three years old, contrasting with the two largest published series, ${ }^{2,6}$ which described a bimodal incidence distribution, with peaks before the age of five years and during adolescence. We also found a bimodal distribution, but with peaks in the 3-5 years and 10-15 years age groups. Unlike some other studies, we found no relationship between young age at presentation and prognosis. ${ }^{2,6,14,20}$ Sex did not influence outcome. As previously reported, ${ }^{14}$ none of our patients was vaccinated or had an allergy shot within 30 days of the first symptoms of ATM, by contrast to a previous center-based analysis of 47 cases. ${ }^{6}$

Idiopathic ATM had the worst prognosis at one year (EDSS $\geq 4$ ), but this difference was not significant at the end of follow-up. In a study of idiopathic ATM in adults with a mean follow-up period of $3.6 \pm 3.1$ years, $30 \%$ of patients had a poor outcome, defined as death or a loss of walking ability. ${ }^{21}$ Consistent with previous reports, ${ }^{6,15}$ we observed potential functional recovery after one year. It remains unclear whether this is due to primary neurological recovery, reeducation or both.

The main finding of this study was the five times higher risk of subsequent MS diagnosis in children with acute partial transverse myelitis at initial presentation. To our knowledge, this is the first study to focus on APTM and MS risk in children. Since 1992, ATM has been divided into two subgroups by several authors: acute complete transverse myelitis (ACTM) and acute partial transverse myelitis (APTM), which differ in terms of clinical presentation, prognosis and course. ${ }^{22-24}$ This clinical dichotomy was highlighted in 2011, in the American Academy of Neurology (AAN) evidence-based guidelines. ${ }^{4}$ The 2002 criteria classified patients into relatively homogeneous groups in terms of clinical and MRI data, but outcome remained unpredictable (except for 'spinal shock' with complete paraplegia). ${ }^{21}$ It should also be noted that all four of our initial clinical scenarios wound up including MS cases. It was therefore necessary to identify other prognostic factors in ATM. In the AAN evidence-based guidelines, a review of two studies of APTM with normal brain MRI in adults reported subsequent diagnosis of MS in $10.3 \%$ of cases $(95 \% \mathrm{CI}$ 4.1-23.6), whereas two studies of adults with ACTM and normal brain MRI suggested a significantly lower subsequent diagnosis of MS ( $0 \%$ to $2 \%$ ), over about five years of follow-up, as in our study. For adults with APTM and cerebral lesions on MRI, the subsequent diagnosis of MS was significantly higher, at 59 to $90 \% .^{4,11,25-28}$ By comparison, subsequent MS diagnosis in our pediatric series was of $20 \%$ for APTM with normal brain MRI, $66 \%$ for APTM with abnormal brain MRI, and 0\% for ACTM. Our results suggest that, as in adults, cerebral MRI status at presentation is the most reliable indicator of the risk of subsequent MS 


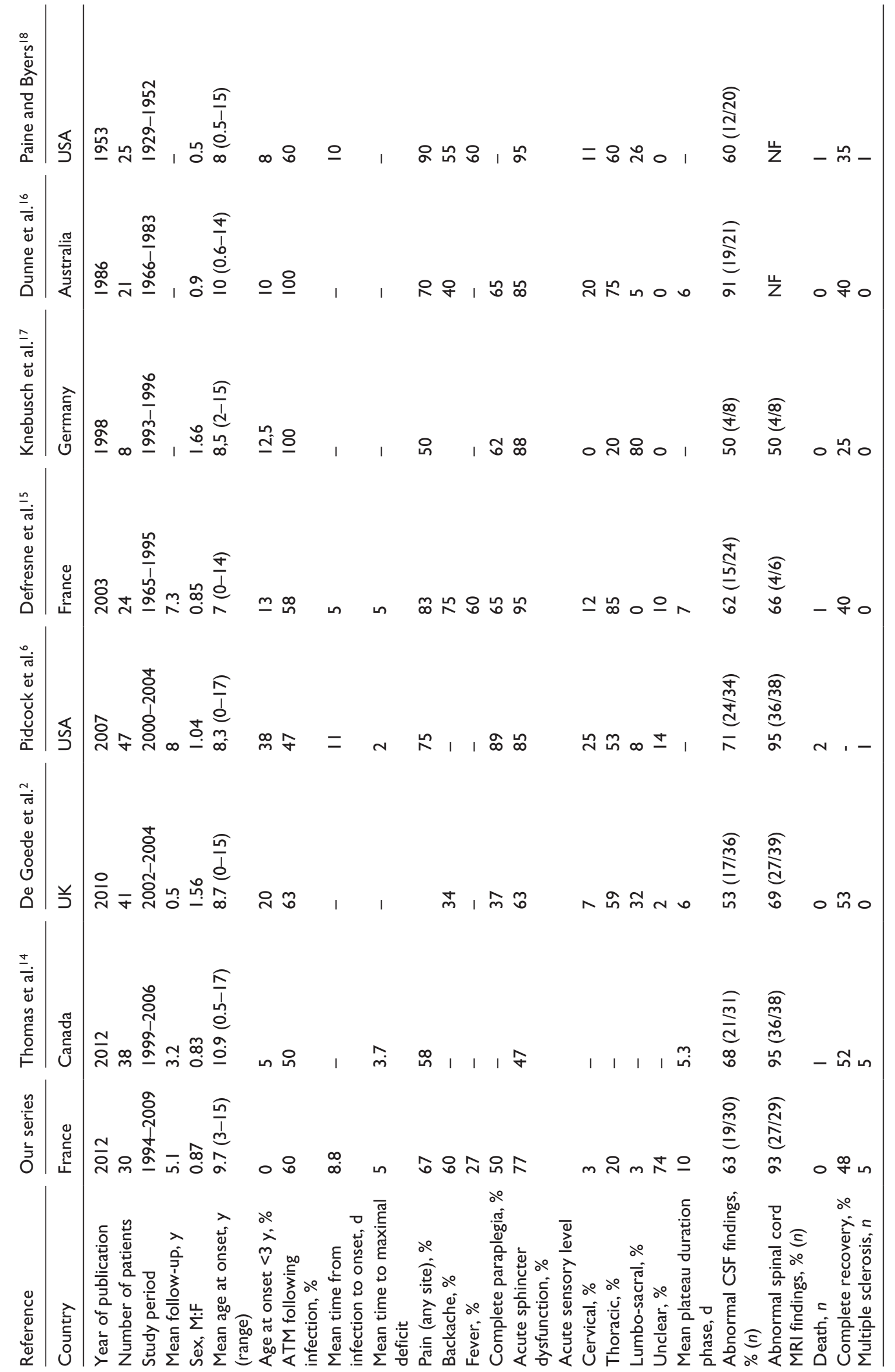


diagnosis in children with APTM. Yearly cerebral MRI for at least five years, with clinical assessments, has been suggested, to monitor adult patients with APTM for MS diagnosis. ${ }^{22}$ Our results support this suggestion, which also appears reasonable for children, to optimize the early diagnosis of MS.

We compared the performances of the 2010 McDonald, Verhey, KIDMUS, and CHAMPS criteria for predicting subsequent diagnosis of MS (see Table 1). Table 1 also shows previous published series studying these criteria, but it is to note that none of them focused specifically on acute transverse myelitis.9,11,29-32 In our series, all criteria had low sensitivity, but high specificity, the highest values being those for the Verhey criteria. These properties have already been reported for children, particularly those older than 10, for KIDMUS criteria. ${ }^{9,10}$ It has also been shown that the 2010 McDonald had high sensitivity and specificity (100\% and $86 \%$, respectively) or children older than 11 years with non-ADEM presentations. ${ }^{30}$ The greatest advantage of the Verhey, 2010 McDonald, and KIDMUS scales was their high negative predictive value. Moreover, the low positive predictive value (PPV) of the 2010 McDonald criteria (50\%) highlighted the need for specific pediatric scales, such as the Verhey criteria, with a higher PPV (80\%). This is the first time that the CHAMPS criteria have been studied in a pediatric population, but they were not found to be particularly useful, particularly given their very low PPV.

Gadolinium infusion demonstrated lesion enhancement on T1W images in 31\% of spinal MRI scans and may explain the low McDonald Dissemination in Time criteria in our cohort. Gadolinium enhancement in pediatric ATM series is of 24 to $74 \%, 6,14$ leading to suggest that the 2002 consortium guidelines may underdiagnose pediatric ATM, but this needs further studies.

One of the five patients manifesting with an ADEM phenotype at onset had two relapses, without encephalopathy, during follow-up and was diagnosed with MS. ADEM-like episode as a first event of MS is reported in 2 to $18 \%$ of cases in published pediatric series of patients with demyelination. ${ }^{9,19,33}$

As reported for both adult and pediatric ATM, a 'spinal shock' presentation (complete paraplegia, hypotonia, and areflexia) was associated with a poor EDSS score at the end of follow-up. 2,15,21,34 However, unlike other studies, we found no relationship between poor prognosis and higher sensitive level, ${ }^{6}$ pyramidal syndrome, ${ }^{20}$ or supraspinal symptoms. ${ }^{15}$ We confirmed that taking more than one month to walk independently was associated with a poor EDSS score at the end of follow-up. ${ }^{15}$

We found no correlation between spinal lesion length and subsequent diagnosis of MS, probably due to our small number of MS cases, or EDSS score at the end of followup. In adult MS patients, the risk of such diagnosis is higher in patients with spinal lesions less than two sections long $^{23}$, but this result has never been confirmed in children. ${ }^{14,15,35,36}$ We found no association between oligoclonal band detection and MS in our series, probably due to the small number of patients tested. Unlike Pidcock et al., ${ }^{6}$ we found no relationship between poor prognosis and lower lesion level and T1 hypointensity on spinal MRI. Most children had LETM, as previously reported, ${ }^{6,36}$ and none had neuromyelitis optica according to the Wingerchuk criteria. $^{7}$ Neuromyelitis optica antibody tests were negative in all four children studied.

\section{Conclusion}

Our single-center analysis of 30 pediatric cases of acute transverse myelitis highlighted the significantly higher risk of multiple sclerosis diagnosis in cases of acute partial transverse myelitis, as previously reported for adults. ${ }^{4}$ This simple clinical dichotomy (mild to moderate weakness, asymmetric or dissociated sensory symptoms), which can be assessed at the patient's bedside during the acute phase, provided valuable clinical decision support. Together with brain MRI data, it could help to identify children at higher risk of subsequent diagnostic of multiple sclerosis, leading to closer brain MRI monitoring or to early disease-modifying treatment.

\section{Acknowledgement}

Dr Pierre Meyer had full access to all of the data in the study and takes responsibility for the integrity of the data and the accuracy of the data analysis.

\section{Conflict of interest}

The authors declare that there is no conflict of interest.

\section{Funding}

This research received no specific grant from any funding agency in the public, commercial, or not-for-profit sectors.[AQ: 1]

\section{References}

1. Banwell B, Kennedy J, Sadovnick D, et al. Incidence of acquired demyelination of the CNS in Canadian children. Neurology 2009; 72: 232-239.

2. De Goede CGEL, Holmes EM, Pike MG. Acquired transverse myelopathy in children in the United Kingdom - a 2 year prospective study. Eur J Paediatr Neurol 2010; 14: 479-487.

3. Group TMCW. Proposed diagnostic criteria and nosology of acute transverse myelitis. Neurology 2002; 59: 499-505.

4. Scott TF, Frohman EM, de Seze J, et al. Evidence-based guideline: clinical evaluation and treatment of transverse myelitis: report of the Therapeutics and Technology Assessment Subcommittee of the American Academy of Neurology. Neurology 2011; 77: 2128-2134.

5. Group TG-BSS. Plasmapheresis and acute Guillain-Barré syndrome. 1985; 35: 1096-1104. 
6. Pidcock FS, Krishnan C, Crawford TO, et al. Acute transverse myelitis in childhood: center-based analysis of 47 cases. Neurology 2007; 68: 1474-1480.

7. Wingerchuk DM, Lennon VA, Pittock SJ, et al. Revised diagnostic criteria for neuromyelitis optica. Neurology 2006; 66: $1485-1489$.

8. Polman CH, Reingold SC, Banwell B, et al. Diagnostic criteria for multiple sclerosis: 2010 revisions to the McDonald criteria. Ann Neurol 2011; 69: 292-302.

9. Verhey LH, Branson HM, Shroff MM, et al. MRI parameters for prediction of multiple sclerosis diagnosis in children with acute CNS demyelination: a prospective national cohort study. Lancet Neurol 2011; 10: 1065-1073.

10. Mikaeloff Y, Adamsbaum C, Husson B, et al. MRI prognostic factors for relapse after acute CNS inflammatory demyelination in childhood. Brain 2004; 127: 1942-1947.

11. Jacobs LD, Beck RW, Simon JH, et al. Intramuscular interferon beta-1a therapy initiated during a first demyelinating event in multiple sclerosis. CHAMPS Study Group. N Engl J Med 2000; 343: 898-904.

12. Kurtzke JF. Rating neurologic impairment in multiple sclerosis: an Expanded Disability Status Scale (EDSS). Neurology 1983; 33: 1444-1452.

13. Krupp LB, Tardieu M, Amato MP, et al. International Pediatric Multiple Sclerosis Study Group criteria for pediatric multiple sclerosis and immune-mediated central nervous system demyelinating disorders: revisions to the 2007 definitions. Mult Scler 2013; 19: 1261-1267.

14. Thomas T, Branson HM, Verhey LH, et al. The demographic, clinical, and magnetic resonance imaging (MRI) features of transverse myelitis in children. $J$ Child Neurol 2012; 27: 11-21.

15. Defresne P, Hollenberg H, Husson B, et al. Acute transverse myelitis in children: clinical course and prognostic factors. $J$ Child Neurol 2003; 18: 401-406.

16. Dunne K, Hopkins IJ and Shield LK. Acute transverse myelopathy in childhood. Dev Med Child Neurol 1986; 28: 198-204.

17. Knebusch M, Strassburg HM and Reiners K. Acute transverse myelitis in childhood: nine cases and review of the literature. Dev Med Child Neurol 1998; 40: 631-639.

18. Paine RS and Byers RK. Transverse myelopathy in childhood. AMA Am J Dis Child 1953; 85: 151-163.

19. Neuteboom RF, Boon M, Catsman Berrevoets CE, et al. Prognostic factors after a first attack of inflammatory CNS demyelination in children. Neurology 2008; 71: 967-973.

20. Miyazawa R, Ikeuchi Y, Tomomasa T, et al. Determinants of prognosis of acute transverse myelitis in children. Pediatr Int 2003; 45: 512-516.
21. de Seze J, Lanctin C, Lebrun C, et al. Idiopathic acute transverse myelitis: application of the recent diagnostic criteria. Neurology 2005; 65: 1950-1953.

22. Scott TF. Nosology of idiopathic transverse myelitis syndromes. Acta Neurol Scand 2007; 115: 371-376.

23. de Seze J, Stojkovic T, Breteau G, et al. Acute myelopathies: clinical, laboratory and outcome profiles in 79 cases. Brain 2001; 124: 1509-1521.

24. Ford B, Tampieri D and Francis G. Long-term follow-up of acute partial transverse myelopathy. Neurology 1992; 42: 250-252.

25. Scott TF, Kassab SL and Singh S. Acute partial transverse myelitis with normal cerebral magnetic resonance imaging: transition rate to clinically definite multiple sclerosis. Mult Scler 2005; 11: 373-377.

26. Morrissey SP, Miller DH, Kendall BE, et al. The significance of brain magnetic resonance imaging abnormalities at presentation with clinically isolated syndromes suggestive of multiple sclerosis. A 5-year follow-up study. Brain 1993; 116: 135-146.

27. Bashir K and Whitaker JN. Importance of paraclinical and CSF studies in the diagnosis of MS in patients presenting with partial cervical transverse myelopathy and negative cranial MRI. Mult Scler 2000; 6: 312-316.

28. Bourre B, Zéphir H, Ongagna J-C, et al. Long-term followup of acute partial transverse myelitis. Arch Neurol 2012; 69: 357-362.

29. Kornek B, Schmitl B, Vass K, et al. Evaluation of the 2010 McDonald multiple sclerosis criteria in children with a clinically isolated syndrome. Mult Scler 2012; 18: 1768-1774.

30. Sadaka Y, Verhey LH, Shroff MM, et al. 2010 McDonald criteria for diagnosing pediatric multiple sclerosis. Ann Neurol 2012; 72: 211-223.

31. Tantsis EM, Prelog K, Brilot F, et al. Risk of multiple sclerosis after a first demyelinating syndrome in an Australian paediatric cohort: clinical, radiological features and application of the McDonald 2010 MRI criteria. Mult Scler 2013; 19: 1749-1759.

32. Young J, Quinn S, Hurrell M, et al. Clinically isolated acute transverse myelitis: prognostic features and incidence. Mult Scler 2009; 15: 1295-1302

33. Mikaeloff Y, Caridade G, Husson B, et al. Acute disseminated encephalomyelitis cohort study: prognostic factors for relapse. Eur J Paediatr Neurol 2007; 11: 90-95.

34. Kalita J, Misra UK and Mandal SK. Prognostic predictors of acute transverse myelitis. Acta Neurol Scand 1998; 98: 60-63.

35. Andronikou S, Albuquerque-Jonathan G, Wilmshurst J, et al. MRI findings in acute idiopathic transverse myelopathy in children. Pediatr Radiol 2003; 33: 624-629.

36. Alper G, Petropoulou KA, Fitz CR, et al. Idiopathic acute transverse myelitis in children: an analysis and discussion of MRI findings. Mult Scler 2011; 17: 74-80. 